\title{
ASYMPTOTIC INTEGRATIONS OF NONOSCILLATORY SECOND ORDER DIFFERENTIAL EQUATIONS
}

\author{
SHAOZHU CHEN
}

\begin{abstract}
The linear differential equation (1) $\left(r(t) x^{\prime}\right)^{\prime}+(f(t)+q(t)) x=0$ is viewed as a perturbation of the equation $(2)\left(r(t) y^{\prime}\right)^{\prime}+f(t) y=0$, where $r>0, f$ and $q$ are real-valued continuous functions. Suppose that (2) is nonoscillatory at infinity and $y_{1}, y_{2}$ are principal, nonprincipal solutions of (2), respectively. Adapted Riccati techniques are used to obtain an asymptotic integration for the principal solution $x_{1}$ of (1). Under some mild assumptions, we characterize that (1) has a principal solution $x_{1}$ satisfying $x_{1}=y_{1}(1+o(1))$. Sufficient (sometimes necessary) conditions under which the nonprincipal solution $x_{2}$ of (1) behaves, in three different degrees, like $y_{2}$ as $t \rightarrow \infty$ are also established.
\end{abstract}

\section{INTRODUCTION}

Consider the second order differential equation

$$
\left(r(t) x^{\prime}\right)^{\prime}+(f(t)+q(t)) x=0
$$

as a perturbation of the equation

$$
\left(r(t) y^{\prime}\right)^{\prime}+f(t) y=0,
$$

where $r, f, q, x$ and $y$ are continuous and real-valued functions on $[a, \infty)$, $r>0$. Suppose that $(1.2)$ is nonoscillatory at infinity. It is well known [3, p. 355] that (1.2) has a principal solution $y_{1}$ which is essentially unique (up to a constant factor) such that

$$
\int^{\infty} d t /\left(r y_{1}^{2}\right)=\infty
$$

and for any solution $y_{2}$ linearly independent of $y_{1}$,

$$
y_{1} / y_{2} \rightarrow 0, \quad t \rightarrow \infty .
$$

The solution $y_{2}$ is called a nonprincipal solution. Without loss of generality, we assume that $y_{1}(t)>0$ for $t \geq a$. Set

$$
g(t)=\int_{a}^{t} d s /\left(r y_{1}^{2}\right)
$$

Received by the editors September 11, 1989.

1980 Mathematics Subject Classification (1985 Revision). Primary 34C99; Secondary 34C10.

Key words and phrases. Nonoscillatory equations, linear perturbations, asymptotic integrations, Riccati techniques. 
then $y_{1} g$ is a nonprincipal solution. We will assume throughout this paper that $y_{2}(t)=y_{1}(t)[g(t)-g(b)]$ for some $b \geq a$ and hence $y_{2}$ is eventually positive and satisfies

$$
r\left(y_{1} y_{2}^{\prime}-y_{1}^{\prime} y_{2}\right)=1 \text {. }
$$

We will be interested in finding conditions on $q$ so that solutions of (1.1) will behave asymptotically like those of (1.2). Some remarks about known results on this seem to be appropriate here.

In what follows, $L$ denotes the set of all continuous functions $z$ on $[b, \infty)$ for some $b \geq a$ such that the improper integrals $\int_{b}^{\infty} z d t$ converge (at least conditionally), $z^{*}(t)=\sup _{s \geq t}|z(s)|$ whenever $z(t)$ is bounded for $t \geq b$ and

$$
\bar{z}(t, b)=[g(t)-g(b)]^{-1} \int_{b}^{t} g^{\prime} z d s,
$$

the weighted average of $z$ over $[b, t]$. We also write $\bar{z}(t)=\bar{z}(t, a)$ for short. The Landau notations " $O$ " and " $O$ " refer to behavior as $t \rightarrow \infty$.

Hartman and Wintner considered the equation (1.1) where $q$ may be complex-valued and obtained

Theorem A [3, p. 379]. If

$$
y_{1}^{2} q \in L
$$

and

$$
\int^{\infty} g^{\prime} Q^{*} d t<\infty
$$

where $Q(t)=\int_{t}^{\infty} y_{1}^{2} q d s$, then (1.1) has solutions $x_{1}$ and $x_{2}$ such that $x_{i}=$ $y_{i}(1+o(1))$ and $x_{i}^{\prime} / x_{i}-y_{i}^{\prime} / y_{i}=o\left(\left(r y_{1} y_{2}\right)^{-1}\right)$ for $i=1,2$.

Theorem A was improved by Trench:

Theorem B [6, Theorem 2]. Suppose that

$$
g y_{1}^{2} q \in L
$$

and $Q_{1}^{*}=O(\phi)$, where $Q_{1}(t)=\int_{t}^{\infty} g y_{1}^{2} q d s, \phi(t)>0$ and $\phi(t) \rightarrow 0$ monotonically as $t \rightarrow \infty$. If

$$
\int^{\infty} g^{\prime}|Q| \phi d t<\infty
$$

and

$$
\limsup _{t \rightarrow \infty}(\phi(t))^{-1} \int_{t}^{\infty} g^{\prime}|Q| \phi d s=A<1 / 3,
$$

then (1.1) has solutions $x_{1}$ and $x_{2}$ such that $x_{1}=y_{1}(1+O(\phi)),\left(x_{1} / y_{1}\right)^{\prime}=$ $O\left(\phi g^{\prime} / g\right), x_{2}=y_{2}(1+O(\bar{\phi}))$ and $\left(x_{2} / y_{2}\right)^{\prime}=O\left(\bar{\phi} g^{\prime} / g\right)$.

Note that $(1.9) \Rightarrow(1.7)$ but $(1.7)-(1.8) \Rightarrow(1.9)-(1.11)$ (see [6]). Šimša [5] proved that $1 / 3$ can be replaced by 1 in (1.11) and obtained 
Theorem C [5, Theorem 1]. If (1.9) holds and

$$
\int^{\infty} g^{\prime}\left|Q_{1}\right| \phi / g d t<\infty
$$

then (1.1) has a solution $x_{1}$ such that $x_{1}=y_{1}\left[1+O\left(\phi+\int_{t}^{\infty} g^{\prime}\left|Q_{1}\right| \phi / g d s\right)\right]$ and $\left(x_{1} / y_{1}\right)^{\prime}=O\left(\phi g^{\prime} / g\right)$.

The approach to proving Theorems $B$ and $C$ in $[6,5]$ is mainly based on finding the principal solutions $x_{1}$ of $(1.1)$ by a fixed point theorem. Instead a different method, the Riccati transformation, will be used here in the case where $q$ is assumed to be real-valued; this method seems to be advantageous to establishing a rather accurate asymptotic integration for the principal solution $x_{1}$ of (1.1) which is asymptotic to $y_{1}$. For the nonprincipal solutions, more asymptotic properties, of interest in their own right, should be specified as follows:

$$
\begin{gathered}
x_{2}=y_{2}(1+o(1)), \\
x_{2}^{\prime} / x_{2}-y_{2}^{\prime} / y_{2}=\Delta=o\left(\left(r y_{1} y_{2}\right)^{-1}\right) \text { and } \Delta \in L, \\
x_{2}-y_{2}=y_{1}(C+o(1)),
\end{gathered}
$$

where $C$ is a constant. Among them, (1.13) is obviously the weakest but (1.14) and (1.15) are independent of each other (Example 4.2). Motivations of investigating asymptotic properties of nonprincipal solutions in greater detail as above can also be found in [1,2], where other kinds of differential equations were discussed.

In this paper, we will obtain sufficient (sometimes necessary) conditions on $q$ under which (1.1) has a principal solution $x_{1}$ satisfying $x_{1}=y_{1}(1+o(1))$ and a nonprincipal solution $x_{2}$ satisfying each of the three asymptotic properties (1.13)-(1.15).

\section{GeNeralized Riccati EQuATIONS AND NONOSCILlation}

Let $x$ be a nonoscillatory solution of $(1.1), x(t) \neq 0$ for $t \geq b$ for some $b \geq a$. Let $u=r x^{\prime} / x$. Then $u$ satisfies

$$
u^{\prime}+u^{2} / r+f+q=0, \quad t \geq b .
$$

Set $u=\eta+v$, where $\eta=r y_{1}^{\prime} / y_{1}$. Then $v$ satisfies

$$
v^{\prime}+2 \eta v / r+v^{2} / r+q=0
$$

or

$$
\left(y_{1}^{2} v\right)^{\prime}+y_{1}^{2} v^{2} / r+y_{1}^{2} q=0, \quad t \geq b .
$$

Equation (2.1) is called the generalized Riccati equation for (1.1) with respect to (1.2). Where $f \equiv 0$ and $y_{1} \equiv 1$, as in [3, pp. 331-332; 7, 8] (2.1) reduces to the ordinary Riccati equation. It is clear that $(1.1)$ is nonoscillatory at infinity if and only if $(2.1)$ has a solution $v(t)$ for $t \geq b$ for some $b \geq a$. Moreover, we can establish the following result involving a generalized Riccati integral equation for (1.1) with respect to (1.2). 
Lemma 2.1. If

$$
\liminf _{t \rightarrow \infty}[g(t)]^{-1} \int_{a}^{t} g^{\prime}(s)\left(\int_{a}^{s} y_{1}^{2} q d \sigma\right) d s>-\infty,
$$

then (1.1) is nonoscillatory if and only if for some $b \geq a$, the limit

$$
\lim _{t \rightarrow \infty}[g(t)-g(b)]^{-1} \int_{b}^{t} g^{\prime}(s)\left(\int_{b}^{s} y_{1}^{2} q d \sigma\right) d s=C_{b}
$$

exists (finite) and there exists a continuous function $v(t)$ for $t \geq b$ such that $y_{1}^{2} v^{2} / r \in L$ and

$$
y_{1}^{2} v=Q(t)+\int_{t}^{\infty} y_{1}^{2} v^{2} / r d s
$$

where

$$
Q(t)=C_{b}-\int_{b}^{t} y_{1}^{2} q d s .
$$

Note that $y_{1}^{2} q \in L \Rightarrow(2.3) \Rightarrow(2.2)$, but in general the converses are not true. However, if $(1.1)$ is nonoscillatory, then $(2.2) \Leftrightarrow(2.3)$. We also observe that if (2.3) holds for some $b_{0} \geq a$ then it holds for all $b \geq a$. Letting $t \rightarrow \infty$ in the following identity

$$
\begin{aligned}
& {[g(t)-g(b)]^{-1} \int_{b}^{t} g^{\prime}(s) \int_{b}^{s} y_{1}^{2} q d \sigma d s} \\
& \quad=[g(t)-g(b)]^{-1}\left\{\int_{a}^{t} g^{\prime}(s) \int_{a}^{s} y_{1}^{2} q d \sigma d s\right. \\
& \left.\quad-\int_{a}^{b} g^{\prime}(s) \int_{a}^{s} y_{1}^{2} q d \sigma d s-\int_{b}^{t} g^{\prime} d s \int_{a}^{b} y_{1}^{2} q d \sigma\right\}
\end{aligned}
$$

gives $C_{b}=C_{a}-\int_{a}^{b} y_{1}^{2} q d s$. Therefore, the function $Q(t)$ given by (2.5) is independent of the values of $b$. Thus in the sequel, by saying that $Q(t)$ exists (finite) we mean that (2.3) holds and $Q(t)$ is defined by (2.5) for $b \geq a$. In particular, if $y_{1}^{2} q \in L$ then $Q(t)=\int_{t}^{\infty} y_{1}^{2} q d s$.

We next introduce a generaltized Riccati equation of the second level. Suppose that (2.2) holds and (1.1) is nonoscillatory. Then $Q(t)$ exists (finite) and $y_{1}^{2} v^{2} / r \in L$. Hence, if we let

$$
w(t)=\int_{t}^{\infty} y_{1}^{2} v^{2} / r d s, \quad t \geq b,
$$

then $w$ satisfies

$$
w^{\prime}+2 g^{\prime} Q w+g^{\prime}\left(w^{2}+Q^{2}\right)=0, \quad t \geq b .
$$

Set $G(s, t)=\exp \left(2 \int_{t}^{s} g^{\prime} Q d \sigma\right)$ and

$$
P(t)=\int_{t}^{\infty} g^{\prime} Q^{2} G(s, t) d s
$$

whenever the integrands make sense and the integrals converge. We now have 
Lemma 2.2. Let (2.2) hold. Then (1.1) is nonoscillatory if and only if $Q(t)$ and $P(t)$ exist (finite) and there exists a continuous function $w(t), t \geq b$ for some $b \geq a$, such that

$$
w(t)=P(t)+\int_{t}^{\infty} g^{\prime} w^{2} G(s, t) d s, \quad t \geq b .
$$

Remark. If (2.2) holds and $x$ is a nonoscillatory solution of (1.1), then the function $w=y_{1}^{2} v-Q$ must satisfy (2.9), where $v=r x^{\prime} / x-\eta$.

The proofs of Lemmas 2.1 and 2.2 are omitted because they can be obtained either by employing the same arguments used in $[7,8]$ for ordinary Riccati equations or by letting $x=y_{1} z, g(t)=s$ and then applying the known results on ordinary Riccati equations to the equation

$$
d^{2} z / d s^{2}+r y_{1}^{4} q z=0
$$

The integral equation (2.9) has the advantage of involving the nonnegative functions $P$ and $w$ but no individual values of $q$. One can easily make use of (2.9) to estimate the function $w$ and hence the nonoscillatory solution $x$ of (1.1). This thought will partially be embodied in the next nonoscillation result (Theorem 2.4). Before giving this result, we introduce a lemma first.

Lemma 2.3. Suppose that $Q(t)$ exists (finite) and $g^{\prime} Q \in L$. Then

$$
\psi(t)=\int_{t}^{\infty} g g^{\prime} Q^{2} d s<\infty
$$

if and only if $P(t)$ exists (finite) and $g^{\prime} P \in L$.

Proof. Since $g^{\prime} Q \in L$, there is an $M>1$ such that

$$
M^{-1} \leq G(s, t) \leq M, \quad t, s \geq a .
$$

For any $T>t$, we have

$$
\begin{aligned}
\int_{t}^{T} g^{\prime} P d s & =\int_{t}^{T} g^{\prime}(s) \int_{s}^{\infty} g^{\prime} Q^{2} G(\sigma, s) d \sigma d s \\
& \leq M \int_{t}^{T} g^{\prime}(s) \int_{s}^{\infty} g^{\prime} Q^{2} d \sigma d s \\
& =M\left(\int_{t}^{T}(g(s)-g(t)) g^{\prime}(s) Q^{2}(s) d s+(g(T)-g(t)) \int_{T}^{\infty} g^{\prime} Q^{2} d s\right) \\
& \leq M \int_{t}^{\infty} g g^{\prime} Q^{2} d s
\end{aligned}
$$

and

$$
\begin{aligned}
\int_{t}^{T} g^{\prime} P d s & \geq M^{-1} \int_{t}^{T} g^{\prime}(s) \int_{s}^{\infty} g^{\prime} Q^{2} d \sigma d s \\
& \geq M^{-1}\left(\int_{t}^{T} g g^{\prime} Q^{2} d s-g(t) \int_{t}^{\infty} g^{\prime} Q^{2} d s\right) .
\end{aligned}
$$

Then Lemma 2.3 follows immediately. 
Theorem 2.4. If $Q(t)$ exists (finite), $g^{\prime} Q \in L$ and $\left(\mathrm{A}_{1}\right)$ holds, then (1.1) is nonoscillatory.

Proof. Our proof proceeds on the basis of constructing a solution of (2.9).

By Lemma 2.3, ( $\left.\mathrm{A}_{1}\right)$ implies that $P$ exists and $g^{\prime} P \in L$. Let $b \geq a$ be so large that for all $t \geq b$,

$$
\int_{t}^{\infty} g^{\prime} P d s<\frac{1}{4} M^{-3}
$$

Let $X$ be the Banach space of all bounded, continuous functions on $[b, \infty)$ equipped with the norm given by $\|z\|=\sup _{t \geq b}\{|z(t)|\}, z \in X$. Let $S=\{z \in$ $X: P(t) \leq z(t) \leq 2 P(t), t \geq b\}$. Then $S$ is a closed convex subset of $X$. Define an operator $T: S \rightarrow X$ by

$$
(T z)(t)=P(t)+\int_{t}^{\infty} g^{\prime} z^{2} G(s, t) d s
$$

for any $z \in S$. Since $g^{\prime} Q \in L$, from (2.10), we have for $s \geq t$,

$$
\begin{aligned}
P(s) & =\int_{s}^{\infty} g^{\prime} Q^{2} G(\sigma, s) d \sigma \leq M \int_{s}^{\infty} g^{\prime} Q^{2} d \sigma \\
& \leq M \int_{t}^{\infty} g^{\prime} Q^{2} d \sigma \leq M^{2} \int_{t}^{\infty} g^{\prime} Q^{2} G(s, t) d s=M^{2} P(t) .
\end{aligned}
$$

From (2.12), (2.13) and (2.11),

$$
P(t) \leq(T z)(t) \leq P(t)+4 M^{3} P(t) \int_{t}^{\infty} g^{\prime} P d s \leq 2 P(t), \quad t \geq b .
$$

Thus, $T: S \rightarrow S$. On the other hand, for every $t \geq b, z_{1}, z_{2} \in S$, we have

$$
\begin{aligned}
\left|\left(T z_{1}\right)(t)-\left(T z_{2}\right)(t)\right| & =\left|\int_{t}^{\infty} g^{\prime}\left(z_{1}+z_{2}\right)\left(z_{1}-z_{2}\right) G(s, t) d s\right| \\
& \leq 4 M\left\|z_{1}-z_{2}\right\| \int_{t}^{\infty} g^{\prime} P d s \leq M^{-2}\left\|z_{1}-z_{2}\right\|,
\end{aligned}
$$

which shows that $T$ is a contraction. Hence, there exists a unique fixed point $w$ of $T$ in $S$, which is a solution of $(2.9)$ on $[b, \infty)$. This completes the proof of Theorem 2.4.

It should particularly be noted at this point that under the hypotheses of Theorem $2.4,(2.9)$ has a unique solution $w$ on $[b, \infty)$ satisfying

$$
P(t) \leq w(t) \leq 2 P(t) .
$$

Solving $r x^{\prime} / x=r y_{1}^{\prime} / y_{1}+(Q+w) / y_{1}^{2}$ for $x$ we get a solution of $(1.1)$

$$
x_{1}(t)=y_{1}(t) \exp (-H(t)-W(t)),
$$

where $W(t)=\int_{t}^{\infty} g^{\prime} w d s$ and

$$
H(t)=\int_{t}^{\infty} g^{\prime} Q d s
$$


Obviously, $x_{1}$ is a principal solution of (1.1). The asymptotic integration (2.15) of $x_{1}$ with $w$ satisfying (2.14) will play a critical role in $\S 3$.

\section{MAIN Results}

Under some mild assumptions, we first give a necessary and sufficient condition for (1.1) to have a principal solution $x_{1}=y_{1}(1+o(1))$ and a nonprincipal solution $x_{2}$ satisfying (1.13).

Theorem 3.1. Let $Q(t)$ exist (finite) and $g^{\prime} Q \in L$.

(i) If $\left(\mathrm{A}_{1}\right)$ holds, then (1.1) has a principal solution $x_{1}$ satisfying

$$
\begin{gathered}
x_{1}=y_{1}\left[1-H+O\left(H^{2}+\psi\right)\right], \\
\left(x_{1} / y_{1}\right)^{\prime}=g^{\prime} Q\left[1-H+O\left(H^{2}+\psi\right)\right]+O\left(g^{\prime} P\right)
\end{gathered}
$$

and a nonprincipal solution $x_{2}$ satisfying

$$
x_{2}=y_{2}\left[1+O\left(\overline{H^{*}}+\bar{\psi}\right)\right]
$$

for some nonprincipal solution $y_{2}$ of (1.2).

(ii) Conversely, if there are solutions $x$ of (1.1) and $y$ of (1.2) such that $x / y \rightarrow 1$ as $t \rightarrow \infty$, then $\left(\mathrm{A}_{1}\right)$ holds.

Proof. (i) If $\left(\mathrm{A}_{1}\right)$ holds, then by Theorem $2.4,(1.1)$ is nonoscillatory and (2.9) has a solution $w$ satisfying (2.14) for $t \geq b$ for some $b \geq a$. Lemma 2.3 implies that $g^{\prime} P \in L$ and, consequently, $g^{\prime} w \in L$. Let $x_{1}$ be defined as in (2.15). It is then easy to verify that $x_{1}$ is a positive solution of $(1.1)$ on $[b, \infty)$. From (2.10) and (2.14),

$$
\begin{aligned}
W(t) & =\int_{t}^{\infty} g^{\prime} w d s \leq 2 \int_{t}^{\infty} g^{\prime} P d s \leq 2 M \int_{t}^{\infty} g^{\prime}(s) \int_{s}^{\infty} g^{\prime} Q^{2} d \sigma d s \\
& \leq 2 M \int_{t}^{\infty} g g^{\prime} Q^{2} d s=2 M \psi(t) .
\end{aligned}
$$

Thus, (3.1) follows from (2.15) and (3.4). Since

$$
\left(x_{1} / y_{1}\right)^{\prime}=\left(x_{1} / y_{1}\right) g^{\prime}(Q+w) \text {, }
$$

(3.2) follows from (3.5), (3.1) and (2.14).

Since $\int^{\infty} d t /\left(r x_{1}^{2}\right)=\infty, x_{1}$ is a principal solution of $(1.1)$. If we set $x_{2}=$ $x_{1} \int_{b}^{t} d s /\left(r x_{1}^{2}\right)$ and $y_{2}=y_{1}[g(t)-g(b)]$, then $x_{2}, y_{2}$ are nonprincipal solutions of $(1.1),(1.2)$, respectively. We note that $\bar{\psi}(t, b)=O(\bar{\psi}(t))$ and $\psi \leq \bar{\psi}$. From (2.15) and (3.1),

$$
\begin{aligned}
x_{2} / y_{2} & =\left(x_{1} / y_{1}\right)[g(t)-g(b)]^{-1} \int_{b}^{t} g^{\prime}\left(y_{1} / x_{1}\right)^{2} d s \\
& =\left(x_{1} / y_{1}\right)[g(t)-g(b)]^{-1} \int_{b}^{t} g^{\prime}[1+2 H(1+o(1))+O(\psi)] d s \\
& =\left(x_{1} / y_{1}\right)\left[1+O\left(\overline{H^{*}}+\bar{\psi}\right)\right] .
\end{aligned}
$$

We then get (3.3) from (3.6) and (3.1). 
(ii) Let $x_{1}$ and $x_{2}$ be principal and nonprincipal solutions of (1.1), respectively. Suppose that $x=A x_{1}+B x_{2}, y=C y_{1}+D y_{2}$ and $x / y \rightarrow 1$. Since neither $x_{1} / y_{2}$ nor $x_{2} / y_{1}$ can tend to a nonzero number, we may assume that either $B=D \neq 0$ or $B=D=0$ and $A=C \neq 0$, and hence either $x_{2} / y_{2} \rightarrow 1$ or $x_{1} / y_{1} \rightarrow 1$. If $x_{2} / y_{2} \rightarrow 1$, then

$$
\lim _{t \rightarrow \infty} x_{1} / y_{1}=\lim _{t \rightarrow \infty}\left(x_{2} / y_{2}\right) \int_{t}^{\infty} d s /\left(r x_{2}^{2}\right)\left[\int_{t}^{\infty} d s /\left(r y_{2}^{2}\right)\right]^{-1}=1
$$

Thus, the assumption of (ii) is reduced to the case $x_{1} / y_{1} \rightarrow 1$.

Assume that $x_{1}(t)>0, t \geq b \geq a$. Let $w=y_{1}^{2} v-Q$, where $v=$ $r x_{1}^{\prime} / x_{1}-r y_{1}^{\prime} / y_{1}$. Since (1.1) is nonoscillatory, by the remark after Lemma 2.2, $w$ satisfies (2.9) and hence $0 \leq P \leq w$. Furthermore,

$$
g^{\prime} w=v / r-g^{\prime} Q=x_{1}^{\prime} / x_{1}-y_{1}^{\prime} / y_{1}-g^{\prime} Q=\left[\ln \left(x_{1} / y_{1}\right)\right]^{\prime}-g^{\prime} Q .
$$

Since $g^{\prime} Q \in L$ and $x_{1} / y_{1} \rightarrow 1$, we have $g^{\prime} w \in L$. As a result, $g^{\prime} P \in L$. Thus $\left(\mathrm{A}_{1}\right)$ holds by the virtue of Lemma 2.3. The proof of Theorem 3.1 is complete.

Remark. In Theorem 3.1 (i), $x_{2}$ and $y_{2}$ have the same value zero at $t=b$. In case $y_{2}=y_{1}[g(t)-g(c)]$ for some $c \neq b$, we still have

$$
x_{2}=y_{2}\left[1+O\left(g^{-1}+\overline{H^{*}}+\bar{\psi}\right)\right] \text {. }
$$

Next, we give necessary and sufficient conditions for the nonprincipal solutions of (1.1) and (1.2) to have the property (1.14).

Theorem 3.2. Suppose that $Q(t)$ exists (finite) and $g^{\prime} Q \in L$.

(i) If $\left(\mathrm{A}_{1}\right)$ holds, and

$$
g Q \rightarrow 0 \text { as } t \rightarrow \infty,
$$

then (1.1) has a nonprincipal solution $x_{2}$ satisfying $x_{2}^{\prime} / x_{2}-y_{2}^{\prime} / y_{2} \in L$ and

$$
x_{2}^{\prime} / x_{2}-y_{2}^{\prime} / y_{2}=\left(r y_{1} y_{2}\right)^{-1}\left[\left(y_{2} / y_{1}\right) Q+O\left(\overline{H^{*}}+\bar{\psi}\right)\right]
$$

for some nonprincipal solution $y_{2}$ of (1.2).

(ii) Conversely, if (1.1) has a solution $x$ such that $x^{\prime} / x-y_{2}^{\prime} / y_{2} \in L$,

$$
x^{\prime} / x-y_{2}^{\prime} / y_{2}=o\left(\left(r y_{1} y_{2}\right)^{-1}\right),
$$

where $y_{2}$ is a nonprincipal solution of (1.2), then $\left(\mathrm{A}_{1}\right)$ and $\left(\mathrm{A}_{2}\right)$ hold.

Proof. (i) By Theorem 3.1, (1.1) has a solution $x_{1}$ satisfying (3.1) and (3.2) and a solution $x_{2}=x_{1} \int_{b}^{t} d s /\left(r x_{1}^{2}\right)$ satisfying (3.3) for $y_{2}=y_{1}[g(t)-g(b)]$. Let $\Delta=x_{2}^{\prime} / x_{2}-y_{2}^{\prime} / y_{2}$. Then $\Delta \in L$ because of (3.1). Moreover, from (3.5), (3.1) and (3.3) we have

$$
\begin{aligned}
\Delta & =x_{1}^{\prime} / x_{1}+\left(r x_{1} x_{2}\right)^{-1}-y_{1}^{\prime} / y_{1}-\left(r y_{1} y_{2}\right)^{-1} \\
& =\left(x_{1} / y_{1}\right)^{\prime}\left(x_{1} / y_{1}\right)^{-1}+\left[\left(y_{1} / x_{1}\right)\left(y_{2} / x_{2}\right)-1\right]\left(r y_{1} y_{2}\right)^{-1} \\
& =g^{\prime}(Q+w)+\left(r y_{1} y_{2}\right)^{-1} O\left(\overline{H^{*}}+\bar{\psi}\right) \\
& =\left(r y_{1} y_{2}\right)^{-1}\left[(g(t)-g(b))(Q+w)+O\left(\overline{H^{*}}+\bar{\psi}\right)\right] .
\end{aligned}
$$


Since

$$
g w \leq 2 g P \leq 2 M g(t) \int_{t}^{\infty} g^{\prime} Q^{2} d s \leq 2 M \int_{t}^{\infty} g g^{\prime} Q^{2} d s=2 M \psi,
$$

we obtain (3.8) from (3.10).

(ii) Since $x^{\prime} / x-y_{2}^{\prime} / y_{2}=\left[\ln \left(|x| / y_{2}\right)\right]^{\prime} \in L$, integrating from $b$ to $\infty$ gives that $|x(t)| / y_{2}(t)$ tends to a positive number as $t \rightarrow \infty$. Then $\left(\mathrm{A}_{1}\right)$ holds by Theorem 3.1(ii). Let $x=A x_{1}+B x_{2}$, where $x_{1}, x_{2}$ are defined as in Theorem 3.1(i). From (3.9),

$$
\begin{aligned}
x_{2}^{\prime} / x_{2}-y_{2}^{\prime} / y_{2} & =\left(x^{\prime} / x-y_{2}^{\prime} / y_{2}\right)+\left(x_{2}^{\prime} / x_{2}-x^{\prime} / x\right) \\
& =o\left(\left(r y_{1} y_{2}\right)^{-1}\right)+A\left[r x_{2}\left(A x_{1}+B x_{2}\right)\right]^{-1} .
\end{aligned}
$$

If $B=0$, then (3.1), (3.3) an (3.12) lead us to

$$
\begin{aligned}
x_{2}^{\prime} / x_{2}-y_{2}^{\prime} / y_{2} & =o\left(\left(r y_{1} y_{2}\right)^{-1}\right)+\left(r x_{1} x_{2}\right)^{-1} \\
& =\left(r y_{1} y_{2}\right)^{-1}\left[\left(y_{1} / x_{1}\right)\left(y_{2} / x_{2}\right)+o(1)\right] \\
& =\left(r y_{1} y_{2}\right)^{-1}[1+o(1)] .
\end{aligned}
$$

Comparing (3.13) and (3.10) yields that $g Q \rightarrow 1$. Consequently,

$$
\int^{\infty} g g^{\prime} Q^{2} d t=\int^{\infty}(g Q)^{2} g^{\prime} / g d t=\infty .
$$

This contradicts $\left(\mathrm{A}_{1}\right)$, and hence $B \neq 0$. It then follows from (3.12) that

$$
x_{2}^{\prime} / x_{2}-y_{2}^{\prime} / y_{2}=\left(r y_{1} y_{2}\right)^{-1}\left[o(1)+O\left(g^{-1}\right)\right] \text {. }
$$

A closer comparison between (3.14) and (3.10) gives that $g Q \rightarrow 0$, i.e. $\left(\mathrm{A}_{2}\right)$ holds. This completes the proof.

Remarks. (i) In the case where $r \equiv 1$ and $f \equiv 0$, Theorems 3.1 and 3.2 improve a result of Hartman and Wintner in [4] where a different method was used. The latter result was extended to a selfadjoint system of second order differential equations by the author in [2] using Riccati techniques.

(ii) The following lemma will show that our conditions used in Theorems 3.1 and 3.2 are weaker than the conditions (1.9), (1.10) used in Theorem B or (1.9), (1.12) used in Theorem C, provided that $q$ is real-valued.

\section{Lemma 3.3.}

(i) $g y_{1}^{2} q \in L \Leftrightarrow y_{1}^{2} q, g^{\prime} Q \in L$ and $\left(\mathrm{A}_{2}\right)$;

(ii) $g^{\prime} Q Q_{1} \in L \Rightarrow\left(\mathrm{A}_{1}\right)$;

(iii) $g^{\prime} Q_{1}^{2} / g \in L \Rightarrow\left(\mathrm{A}_{1}\right)$.

Proof. Suppose that $g y_{1}^{2} q \in L$. As was indicated in [6], $g y_{1}^{2} q \in L \Rightarrow y_{1}^{2} q \in L$ and hence $Q(t)=\int_{t}^{\infty} y_{1}^{2} q d s$. Consequently,

$$
g Q=-g \int_{t}^{\infty} Q_{1}^{\prime} / g d s=Q_{1}+g(t) \int_{t}^{\infty} Q_{1} d\left((g(s))^{-1}\right) \rightarrow 0 \quad \text { as } t \rightarrow \infty .
$$


Integration by parts produces

Letting $T \rightarrow \infty$, we then have

$$
\int_{t}^{T} g y_{1}^{2} q d s=g(t) Q(t)-g(T) Q(T)+\int_{t}^{T} g^{\prime} Q d s .
$$

$$
Q_{1}=g Q+H,
$$

that is, $g^{\prime} Q \in L$. The sufficiency of the part (i) is clear. Therefore, the proof of (i) is complete.

It follows from (3.15) that

$$
\begin{gathered}
\int_{t}^{\infty} g^{\prime} Q Q_{1} d s=\int_{t}^{\infty} g g^{\prime} Q^{2} d s+\frac{1}{2} H^{2} \\
\int_{t}^{\infty} g^{\prime} Q_{1}^{2} / g d s=\int_{t}^{\infty} g g^{\prime} Q^{2} d s+H^{2}+\int_{t}^{\infty} g^{\prime} H^{2} / g d s .
\end{gathered}
$$

We then get (ii) and (iii) in view of (3.16) and (3.17), respectively.

For (1.1) to have a solution $x_{2}$ satisfying the property (1.15), more assumptions are required.
$\left(\mathrm{A}_{3}\right)$
$g g^{\prime} Q \in L$.
$\left(\mathrm{A}_{4}\right)$

$$
\Psi(t)=\int_{t}^{\infty} g^{2} g^{\prime} Q^{2} d s<\infty .
$$

The following result is similar to Lemma 3.3(i) and hence its proof is omitted. Lemma 3.4. If $Q(t)$ exists, then $\left(\mathrm{A}_{3}\right)$ holds if and only if $g^{\prime} Q \in L, g^{\prime} H \in L$ and $g H \rightarrow 0$ as $t \rightarrow \infty$, in which case

$$
\int_{t}^{\infty} g g^{\prime} Q d s=g H+R
$$

where $R(t)=\int_{t}^{\infty} g^{\prime} H d s$.

It is evidently true that $\left(A_{4}\right) \Rightarrow\left(A_{1}\right)$. Meanwhile, we have more implications of $\left(\mathrm{A}_{4}\right)$.

Lemma 3.5. If $Q(t)$ exists and $\left(\mathrm{A}_{4}\right)$ holds, then for any $\gamma, 0 \leq \gamma<\frac{1}{2}$, we have

$$
\int^{\infty} g^{\gamma} g^{\prime}|Q| d t<\infty
$$

Proof. By Schwarz's inequality, from $\left(\mathrm{A}_{4}\right)$ we have

$$
\left(\int^{\infty} g^{\gamma} g^{\prime}|Q| d t\right)^{2} \leq \int^{\infty} g^{2 \gamma-2} g^{\prime} d t \int^{\infty} g^{2} g^{\prime} Q^{2} d t<\infty
$$

for $\gamma<\frac{1}{2}$. This completes the proof.

Lemma 3.6. If $Q(t)$ exists and $g^{\prime} Q \in L$, then $\left(\mathrm{A}_{4}\right)$ holds if and only if $g g^{\prime} P \in$ $L$.

The proof of Lemma 3.6 follows the steps of that of Lemma 2.3 and is therefore omitted. 
Theorem 3.7. Let $Q(t)$ exist (finite). If $\left(\mathrm{A}_{3}\right)$ and $\left(\mathrm{A}_{4}\right)$ hold, then (1.1) has a principal solution $x_{1}$ satisfying (3.1) and a nonprincipal solution $x_{2}$ such that

$$
x_{2}-y_{2}=y_{1}\left[C-2 R-g H+O\left(H^{*}+\Psi\right)\right]
$$

for some nonprincipal solution $y_{2}$ of (1.2) and some constant $C$.

Proof. Since $\left(\mathrm{A}_{4}\right) \Rightarrow\left(\mathrm{A}_{1}\right)$ and $\left(\mathrm{A}_{3}\right) \Rightarrow g^{\prime} Q \in L$ (Lemma 3.4), Theorem 3.1 provides a principal solution $x_{1}$ of (1.1) satisfying (2.15) and (3.1) for $t \geq b \geq a$. Set $x_{2}=x_{1} \int_{b}^{t} d s /\left(r x_{1}^{2}\right)$ and $y_{2}=y_{1}[g(t)-g(b)]$. From (2.15),

$$
x_{2}=y_{1} e^{-H-W} \int_{b}^{t} g^{\prime} e^{2 H+2 W} d s
$$

and hence

$$
\left(x_{2}-y_{2}\right) / y_{1}=\left(e^{-H-W}-1\right) \int_{b}^{t} g^{\prime} d s+e^{-H-W} \int_{b}^{t} g^{\prime}\left(e^{2 H+2 W}-1\right) d s .
$$

From (3.4) and $g W \leq 2 M g \psi \leq 2 M \Psi$, we have

$$
\left(e^{-H-W}-1\right) \int_{b}^{t} g^{\prime} d s=-g H+O\left(g H^{2}+\Psi\right)+O(|H|) .
$$

We claim that $g^{\prime}\left(e^{2 H+2 W}-1\right) \in L$. To this end, let $e^{2 H}=1+2 H+2 H h$. Then $h=O(H)$ and

$$
g^{\prime}\left(e^{2 H+2 W}-1\right)=2 g^{\prime} H+2 g^{\prime} H h+g^{\prime} O(\psi) .
$$

By Lemma 3.4, $\left(\mathrm{A}_{3}\right) \Rightarrow g^{\prime} H \in L$ and $g H \rightarrow 0$. Also, $\left(\mathrm{A}_{4}\right) \Rightarrow g^{\prime} \psi \in L$ and $\int_{t}^{\infty} g^{\prime} \psi d s=O(\Psi(t))$. It then remains to show that $g^{\prime} H h \in L$. In fact, since $(H h)^{\prime}=-2 g^{\prime} Q H(1+h), g H \rightarrow 0$ and $g^{\prime}|Q| \in L$ (Lemma 3.5), it follows after some manipulation that

$$
\begin{aligned}
\int_{t}^{\infty} g^{\prime} H h d s & =-g H h+2 \int_{t}^{\infty} g g^{\prime} Q H d s+2 \int_{t}^{\infty} g^{\prime} Q g H h d s \\
& =-g H h+g H^{2}+\int_{t}^{\infty} g^{\prime} H^{2} d s+o\left(g H^{2}\right)=O\left(g H^{* 2}\right) .
\end{aligned}
$$

This asserts our claim. Now, if we let $C=\int_{b}^{\infty} g^{\prime}\left(e^{2 H+2 W}-1\right) d s$, then by (3.21)-(3.24) we are led to

$$
\begin{aligned}
\left(x_{2}-y_{2}\right) / y_{1}= & -g H+O\left(g H^{2}+\Psi\right)+e^{-H-W}\left[C-2 R+O\left(g H^{* 2}+\Psi\right)\right] \\
& +O(|H|) \\
= & -g H+O\left(g H^{2}+\Psi\right)+C-C H+O\left(H^{2}+\Psi\right) \\
& -2 R+O\left(g H^{* 2}\right)+O(|H|) \\
= & C-g H-2 R+O\left(H^{*}+\Psi\right),
\end{aligned}
$$

which proves (3.20) and completes the proof.

In view of Theorem 3.7, if $\left(\mathrm{A}_{3}\right)$ and $\left(\mathrm{A}_{4}\right)$ hold, then for any solution $x$ of (1.1) there is a unique solution $y$ of (1.2) for which $x-y=o\left(y_{1}\right)$. In fact, if 
$x=A x_{1}+b x_{2}$, then $y=(A+B C) y_{1}+B y_{2}$, where $C$ is the constant in (3.20). Even so, in case $y_{1}$ is unbounded, $x$ is still in the usual sense not asymptotic to $y$. In general, if $y_{1}$ is unbounded, stronger conditions than $\left(\mathrm{A}_{3}\right)$ and $\left(\mathrm{A}_{4}\right)$ are required so that for any given solution $x$ of (1.1), (1.2) has a solution $y$ such that $x-y=o(1)$. But we will not discuss this problem here. Another thing that should be mentioned is that we have not assumed $\left(\mathrm{A}_{2}\right)$ in Theorem 3.7 and therefore we are not able to ascertain the property (1.14) of the derivatives of nonprincipal solutions. However, this property can be obtained by assuming $\left(\mathrm{A}_{2}\right)$ additionally (see Example 4.2).

\section{EXAMPLES}

To indicate the scope of our results in $\S 3$, we give two examples in this section. Example 4.1. Trench [6] and Šimša [5] have considered the equation

$$
x^{\prime \prime}+K\left[t^{-\beta}(\ln t)^{-\alpha} \sin t\right] x=0
$$

as a perturbation of $y^{\prime \prime}=0$, where $K, \alpha, \beta \neq 0, y_{1}=1$ and $y_{2}=g=t$. Here we assume that $K$ is a real constant. If $\beta=1$, then Theorem B does not apply unless $\alpha \geq 1$ and Theorem $C$ implies that (4.1) has a solution $x_{1}$ satisfying $x_{1}=1+O\left((\ln t)^{-\alpha}+(\ln t)^{1-2 \alpha}\right), x_{1}^{\prime}=O\left(t^{-1}(\ln t)^{-\alpha}\right)$, provided $\alpha>$ $\frac{1}{2}$. For $\alpha>\frac{1}{2}$,

$$
\begin{aligned}
& Q(t)=K t^{-1}(\ln t)^{-\alpha}\left[\cos t+O\left(t^{-1}\right)\right], \\
& H(t)=-K t^{-1}(\ln t)^{-\alpha}\left[\sin t+O\left(t^{-1}\right)\right], \\
& P(t)=O\left(t^{-1}(\ln t)^{-2 \alpha}\right), \\
& \psi(t)=O\left((\ln t)^{1-2 \alpha}\right)=\bar{\psi}(t), \overline{H^{*}}=o(\bar{\psi}),
\end{aligned}
$$

and $\left(A_{1}\right),\left(A_{2}\right)$ hold if and only if $\alpha>\frac{1}{2}$. Then Theorems 3.1 and 3.2 imply that if and only if $\alpha>\frac{1}{2}$, the equation (4.1) has two solutions $x_{1}$ and $x_{2}$ satisfying

$$
\begin{aligned}
& x_{1}=1+K t^{-1}(\ln t)^{-\alpha} \sin t+O\left((\ln t)^{1-2 \alpha}\right), \\
& x_{1}^{\prime}=K t^{-1}(\ln t)^{-\alpha} \cos t+O\left(t^{-1}(\ln t)^{-2 \alpha}\right), \\
& x_{2}=t\left[1+O\left((\ln t)^{1-2 \alpha}\right)\right], \\
& x_{2}^{\prime}=1+K(\ln t)^{-\alpha} \cos t+O\left((\ln t)^{1-2 \alpha}\right) .
\end{aligned}
$$

If $\beta=\frac{3}{2}, \alpha>\frac{1}{2}$, then $\left(\mathrm{A}_{4}\right)$ holds, $\Psi(t)=O\left((\ln t)^{1-2 \alpha}\right), g H=o(\Psi)$ and $R=o(\Psi)$. Thus, Theorem 3.7 implies that

$$
x_{2}-t=C+O\left((\ln t)^{1-2 \alpha}\right),
$$

where $C$ is a constant. Finally, if $\beta=3$ and $\alpha$ is an arbitrary number, then $\Psi(t)=O\left(t^{-3}(\ln t)^{-2 \alpha}\right), H^{*}=O\left(t^{-3}(\ln t)^{-\alpha}\right)$ and so

$$
x_{2}-t=C+K t^{-2}(\ln t)^{-\alpha}\left[\sin t+2 t^{-1} \cos t\right]+O\left(t^{-3}(\ln t)^{-\gamma}\right),
$$

where $\gamma=\min \{\alpha, 1-2 \alpha\}$. 
Example 4.2. Let $\omega_{n}(t)=4(-1)^{n} \sin (n \pi t)$ for $n \leq t \leq n+(1 / n)$ and $\omega_{n}(t)=0$ otherwise, $n=1,2, \ldots$. Let $Q(t)=t^{-\beta}(\ln t)^{-\alpha} \sum_{n=1}^{\infty} \omega_{n}(t), \beta>0$ and $q=-Q^{\prime}$. Consider the equation

$$
x^{\prime \prime}+q(t) x=0 \text {. }
$$

Observing that $\int_{1}^{\infty} \omega_{n}^{2} d t=6 / n, t Q^{2}(t)=t^{1-2 \beta}(\ln t)^{-2 \alpha} \sum \omega_{n}^{2}(t)$, we can easily see that $\left(\mathrm{A}_{1}\right)$ holds if $\beta>\frac{1}{2}$, in which case Theorem 3.1 says that (4.2) has solutions $x_{1}=1+o(1), x_{2}=t+o(t)$. But $\left(\mathrm{A}_{2}\right)$ does not hold unless $\beta>1$ or $\beta=1, \alpha>0$. Thus, either of Theorems $\mathrm{B}$ and $\mathrm{C}$ does not apply for $\beta<1$, because $t q \notin L$ by Lemma 3.3(i). If $\beta>1$ or $\beta=1$ and $\alpha>\frac{1}{2}$, then $\left(\mathrm{A}_{4}\right)$ and $\left(\mathrm{A}_{2}\right)$ hold and so $x_{2}-t \rightarrow$ constant and $x_{2}^{\prime} \rightarrow 1$ as $t \rightarrow \infty$.

However if we choose $Q(t)=t^{-\beta} \sum_{n=1}^{\infty} \omega_{n^{2}}(t)$ instead, then $\left(\mathrm{A}_{4}\right)$ holds for $\beta>\frac{1}{2}$ while $\left(\mathrm{A}_{2}\right)$ does not hold unless $\beta>1$. In other words, for $\frac{1}{2}<\beta \leq 1$, although every solution $x$ of (4.2) is asymptotic to a certain straight line $A+B t(x(t)=A+B t+o(1))$, the derivative of $x$ diverges as $t \rightarrow \infty$. This example supports such a fact that the asymptotic property (1.14) does not have to do with the property (1.15) and therefore both of them should be classified.

\section{REFERENCES}

1. S. Chen, Asymptotic linearity of solutions of nonlinear differential equations, Bull. Austral. Math. Soc. 35 (1987), 257-265.

2. _ Asymptotic linearity of solutions of self-adjoint systems of second order differential equations, J. Math. Anal. Appl. 148 (1990), 509-527.

3. P. Hartman, Ordinary differential equations, Wiley, New York, 1964.

4. P. Hartman and A. Wintner, On non-oscillatory linear differential equations, Amer. J. Math. 75 (1953), 717-730.

5. J. Šimša, Asymptotic integration of a second order ordinary differential equation, Proc. Amer. Math. Soc. 101 (1987), 96-100.

6. W. F. Trench, Linear perturbations of a nonoscillatory second order equation, Proc. Amer. Math. Soc. 97 (1986), 423-428.

7. D. Willett, Classification of second order linear differential equations with respect to oscillation, Adv. in Math. 3 (1969), 594-623.

8. J. S. W. Wong, Oscillation and nonoscillation of solutions of second order linear differential equations with integrable coefficients, Trans. Amer. Math. Soc. 144 (1969), 197-215.

Department of Mathematics, Shandong University, Jinan, Shandong 250100, People's REPUBLIC OF CHINA 